\title{
Funktionalitäten von Essstörungen - Ergebnisse einer Analyse im stationären Setting
}

\author{
Susanne Mikschla ${ }^{a}$ Sandra Schlegl ${ }^{b}$ Anne Katrin Külz ${ }^{c} \quad$ Winfried Rief ${ }^{d} \quad$ Ulrich Voderholzer $^{a, c}$ \\ a Schön Klinik Roseneck, Prien am Chiemsee, Deutschland; \\ ${ }^{b}$ Klinik für Psychiatrie und Psychotherapie, Universitätsklinikum, LMU München, München, Deutschland; \\ ${ }^{\mathrm{c}}$ Abteilung für Psychiatrie und Psychotherapie, Universitätsklinikum Freiburg, Freiburg, Deutschland; \\ ${ }^{d}$ AG Klinische Psychologie und Psychotherapie, Philipps-Universität Marburg, Marburg, Deutschland
}

\section{Schlüsselwörter}

Essstörungen - Anorexia nervosa - Bulimia nervosa . Intrapsychische und interpersonelle Funktionalitäten . Emotionsregulation · Kognitive Verhaltenstherapie

\section{Zusammenfassung}

Einleitung: Symptome psychischer Störungen können verschiedene Funktionalitäten aufweisen, sodass die Symptomatik von Betroffenen häufig nicht ausschließlich als aversiv empfunden wird und eine ambivalente Einstellung gegenüber der Therapie besteht. Ziel der vorliegenden Studie war es, die für die Patienten relevanten intrapsychischen und interpersonellen Funktionalitäten der Essstörung zu erheben. Methode: Mittels eines Pilotfragebogens wurden Funktionalitäten bei Essstörungen an 37 stationären Patientinnen mit Anorexia nervosa (AN) oder Bulimia nervosa (BN) erhoben. Dieser umfasste eine offene Einleitungssowie Abschlussfrage und die Bewertung von 18 vorgegebenen Funktionalitäten. Die Freitextantworten wurden mittels inhaltsanalytischer Techniken kategorisiert sowie über Häufigkeitsverteilungen erfasst. Weiterhin wurden Mittelwerte berechnet und AN mit BN verglichen. Ergebnisse: Essstörungen wird häufiger eine intrapsychische als eine interpersonelle Funktionalität zugeschrieben. Vor allem dienen sie der Emotionsregulation, dem Erlangen von Sicherheit und Kontrolle sowie dem Herstellen von Inhalt und Struktur. Nach der Einleitungsfrage stellt das Herstellen von Nähe und Geborgenheit die häufigste interpersonelle Funktionalität dar; gemäß der Abschlussfrage dienen Essstörungen am häufigsten als Hilferuf. Der einzige signifikante Unterschied bezüglich der Funktionalitäten zwischen Patientinnen mit $A N$ und $B N$ fand sich zur Essstörung als Hilferuf. Schlussfolgerungen: Es konnte eine Vielzahl von Funktionalitäten bei Essstörungen erfasst werden. Intrapsychischen Funktionalitäten kommt eine besondere Relevanz zu, vor allem der Emotionsregulation. Die Ergebnisse unterstreichen die Bedeutung der gezielten Bearbeitung der Funktionalitäten in der störungsspezifischen Psychotherapie.

(c) 2016 S. Karger GmbH, Freiburg

\author{
Keywords \\ Eating disorders - Anorexia nervosa - Bulimia nervosa . \\ Intrapsychic and interpersonal functions . \\ Regulation of emotions - Cognitive behavioral treatment
}

\section{Summary}

Introduction: Symptoms of mental disorders can serve different functions. The affected person frequently does not perceive the symptomatology as solely aversive; thus, the attitude towards therapy can be ambivalent. This survey aimed at identifying intrapsychic and interpersonal functions of eating disorders (EDs). Method: Functions of EDs were analyzed for 37 in-patients with anorexia nervosa (AN) or bulimia nervosa (BN), using a pilot questionnaire that consisted of an open introductory and concluding question each and an assessment of 18 defined functions. Qualitative content analysis was applied to classify the free-text answers. Frequency distributions were calculated, and mean values for AN and BN were compared. Results: Intrapsychic functions were more frequently ascribed to EDs than interpersonal functions. The most prominent functions were emotion regulation, gaining safety and control, and establishing content and structure. The introductory question returned establishing closeness and a feeling of security as the most frequently mentioned interpersonal functions; the answers to the concluding question suggest that EDs serve as a cry for help. The only significant difference between patients with AN and BN with regard to the functions of EDs was ED as a cry for help. Conclusions: A multitude of functions of EDs were registered. Intrapsychic functions, and particularly emotion regulation, are highly relevant. The results highlight the importance of specifically targeting functions in disorder-specific psychotherapy.

\section{KARGER () 2016 S. Karger GmbH, Freiburg 


\section{Einleitung}

«When asked to characterize their reactions to weight loss, anorexics report that they feel delighted, inspired, triumphant, proud and powerful [...] special, superior and deserving of the respect and admiration of others» [Bemis, 1986; zitiert nach Vitousek und Ewald, 1993].

Nach den Kriterien des Diagnostic and Statistic Manual of Mental Disorders (DSM)-5 liegt für Frauen die Lebenszeitprävalenz der Anorexia nervosa (AN) bei bis zu 4\% und der Bulimia nervosa (BN) bei bis zu 2\% [Smink et al., 2013]. Nach der Victorian Burden of Disease Study aus dem Jahr 2001 [Victorian Government Department of Human Services, 2011] sind Essstörungen die vierthäufigste Ursache für eine gesundheitliche Funktionseinschränkung von Frauen in der Adoleszenz und im jungen Erwachsenenalter. Frauen in der relevanten Altersgruppe vom 15. bis 35 . Lebensjahr erleben durchschnittlich 2,125 DALYs (disability-adjusted life years) [Victorian Government Department of Human Services, 2011]. Obwohl dies für die Betroffenen ein hohes Maß an subjektivem Leid bedeuten muss, sind Patienten mit AN oder BN der Therapie gegenüber häufig ambivalent eingestellt [vgl. Garner und Bemis, 1982; Geller et al., 2001; Treasure und Schmidt, 2001; Vitousek et al., 1998]. Wieso aber halten Betroffene an ihren Verhaltensmustern fest, die objektiv betrachtet nichts Positives mit sich bringen und zu Leiden und Beeinträchtigung führen?

Auf die Frage nach der Entstehung und Aufrechterhaltung von Essstörungen findet man in der Literatur bislang keine einstimmige Antwort. Es wird von einem Zusammenspiel verschiedener biologischer, persönlichkeitsbedingter, soziokultureller und familiärer Prädispositions- bzw. Vulnerabilitätsfaktoren ausgegangen, die zur Entstehung einer AN oder BN beitragen. In Bezug auf die Aufrechterhaltung von Essstörungen postulieren beispielsweise Schmidt und Treasure [2006] in ihrem Modell 4 Hauptfaktoren: Perfektionismus, Reaktionen von nahestehenden Personen, die Tendenz, unangenehme innere Erlebnisse zu vermeiden, sowie pro-anorektische Einstellungen. Hier wird also davon ausgegangen, dass Betroffene ihre Erkrankung nicht ausschließlich nachteilig sehen und dass das Maß, in dem sie ihre Störung positiv bewerten, dazu beiträgt, dass die Betroffenen an ihr festhalten.

Umstände, Faktoren und Bedingungen, «die der Symptomatik in ihrem jeweiligen Lebens- und Erlebenskontext eine Sinnhaftigkeit geben», werden nach Külz et al. [2010, S. 102] als Funktionalität bezeichnet. Der Begriff Funktion meint ursprünglich die «Aufgabe, innerhalb eines Ganzen in einer bestimmten Weise wirksam tätig zu sein» [DWDS, 2003]. In der Tiefenpsychologie werden seelisch bedingte Erkrankungen als Lösungsversuche der Psyche betrachtet. Auch hier wird also davon ausgegangen, dass psychische Störungen eine bestimmte Aufgabe für den Betroffenen erfüllen. Diese wird häufig als Krankheitsgewinn bezeichnet; hierbei werden 2 verschiedene Arten unterschieden: primärer und sekundärer Krankheitsgewinn. Unter Ersterem versteht man die Reduktion seelischer Konfliktspannung, während man von sekundärem Krankheitsgewinn spricht, wenn die Betroffenen Vorteile aus ihrer Krankenrolle ziehen [Mentzos, 2009]. Nah an das psychoanalytische Konzept des primären und sekundären Krankheitsgewinns angelehnt ist die Aufteilung in intrapsychische und interpersonelle
Funktionalitäten. Nach Hand [2002] definieren sich diese folgendermaßen:

- Intrapsychische Funktionalitäten: Psychische Symptome haben die Funktion, meist unangenehme intrapsychische Zustände, wie z.B. innere Unruhe, Angst oder Anspannung, aufzuheben oder $\mathrm{zu}$ verringern $\rightarrow$ Geschehen spielt sich innerhalb der Person ab.

- Interpsychische/interpersonelle Funktionalitäten: Psychische Symptome haben die Funktion, ein für den Patienten adäquates Gleichgewicht mit der Umwelt herzustellen $\rightarrow$ Geschehen spielt sich zwischen 2 oder mehr Personen ab.

In der Praxis hat ein Verhalten selten nur eine Funktion, vielmehr sind diese meist komplex miteinander verwoben [Owens und Ashcroft, 1982]. Auch können die gleichen Störungen bei unterschiedlichen Menschen unterschiedliche Funktionen erfüllen [Owens und Ashcroft, 1982].

Die Identifizierung und eingehende Betrachtung der jeweiligen Funktionalität einer psychischen Störung im Lebenskontext des Patienten wird von verschiedenen Therapierichtungen als wichtiger Bestandteil der Diagnostik und Behandlung gesehen. Nach Garner und Bemis [1982] stellt die Bearbeitung der Funktionalität im Laufe der Therapie einen entscheidenden Faktor für den Aufbau von Therapiemotivation, vor allem aber auch für die Aufrechterhaltung der Therapieerfolge dar. Die Analyse der Funktionalitäten von Störungen ist weiterhin ein wesentliches Element der Therapieplanung. Wichtig ist es hierbei, nicht nur die Symptome zu behandeln, sondern auch die nun fehlenden Funktionen [Owens und Ashcroft, 1982] zu bearbeiten, um eventuelle Rückfälle zu vermeiden.

$\mathrm{Zu}$ den bisherigen Therapiemanualen zur Behandlung von Essstörungen, die Module zur Bearbeitung der aufrechterhaltenden Faktoren beinhalten, gehören beispielsweise das Maudsley Model of Treatment of Adults with AN (MANTRA) nach Schmidt et al. [2014], das auf dem obengenannten Modell [Schmidt und Treasure, 2006; Treasure und Schmidt, 2013] beruht, oder das Manual zur fokalen psychodynamischen Psychotherapie der AN [Friederich et al., 2014]. Auch hier ist etwa die «Thematisierung proanorektischer Überzeugungen» Bestandteil der Therapie. Den Betroffenen wird weiterhin die Aufgabe gestellt, einen Brief an ihre Magersucht zu verfassen. Das kognitiv-verhaltenstherapeutische Behandlungsmanual von Salbach-Andrae et al. [2010] für Jugendliche beinhaltet dieses Therapieelement ebenfalls. Eingehend setzt sich auch die erweiterte kognitive Verhaltenstherapie (enhanced cognitive behavior therapy; CBT-E) von Christopher Fairburn [Fairburn, 2008, 2012] mit den aufrechterhaltenden Faktoren auseinander. Hier werden die Vor- und Nachteile einer Verhaltensänderung thematisiert.

Külz [2014] fasst 4 zentrale Aspekte zusammen, die sich aus der Bearbeitung der Funktionalität für die Behandlung ableiten lassen: Erweiterung der Perspektive und Problemeinsicht, Emotionale Entlastung, Identifikation relevanter Therapieziele, Erhöhung der Compliance und Vorbeugung von Rückfällen. Dennoch gerät dieser Aspekt in der klinischen Praxis bisweilen in den Hintergrund. Häufig wird der Fokus primär auf die negativen Konsequenzen der Krankheit, wie etwa gesundheitliche Folgeschäden und zwischen- 
menschliche Probleme, gelegt. Die Frage, was es den Betroffenen so schwermacht, die Störung aufzugeben, bleibt oft ungestellt.

Auch die klinische Erfahrung zeigt, dass die differenzierte Betrachtung von Funktionalitäten für eine erfolgreiche Behandlung psychischer Störungen dringend notwendig ist. Zum einen zeigt sich, dass unbearbeitete Funktionalitäten ein wesentlicher Grund für unzureichende Behandlungsfortschritte auf symptomatischer Ebene sein können, da bewusste oder unbewusste Widerstände einer Besserung entgegenstehen. Zum anderen kann die Identifikation von Funktionalitäten gerade auch den Betroffenen das Verständnis ihrer Erkrankung erleichtern, wichtige Hinweise für die Definition störungsübergreifender Therapieziele geben und die Behandlungsmotivation verbessern.

Eine Sichtung der Literatur zeigt, dass eine systematische und explizite Untersuchung der Funktionalitäten von Essstörungen trotz der hohen klinischen Relevanz bisher vernachlässigt wurde. Serpell et al. [1999] analysierten Briefe, die Patienten mit AN im therapeutischen Prozess an ihre Essstörung zu schreiben hatten, hinsichtlich der subjektiv wahrgenommenen Vor- bzw. Nachteile der Störung. Darauf aufbauend entwickelten sie die Pros and Cons of Anorexia Nervosa (P-CAN)-Skala [Serpell et al., 2004]. Der Einsatz dieses Fragebogens ermöglicht es somit, Rückschlüsse auf die aufrechterhaltenden Bedingungen zu ziehen. Das Messinstrument weist gute psychometrische Gütekriterien auf und wurde erfolgreich bei Erwachsenen, Jugendlichen und Kindern eingesetzt [Serpell et al., 2003]. Darauf aufbauend wurde inzwischen ein Fragebogen zur Erfassung der Vor- und Nachteile von Essstörungen im Allgemeinen entwickelt [Gale et al., 2006]. Eine deutschsprachige Version wurde durch von Brachel et al. [eingereicht] vorgelegt.

Während die Arbeitsgruppe um Serpell sowohl die von Betroffenen als positiv als auch die als negativ wahrgenommenen Aspekte bei Essstörungen betrachtete, konzentriert sich die vorliegende Studie ausschließlich auf die subjektiven Vorteile der Krankheit. Diese sollen noch näher beleuchtet und differenzierter betrachtet werden, da sie zur Aufrechterhaltung der Störung beitragen und vor allem auch in der klinischen Praxis, wie schon oben beschrieben, zum Teil vernachlässigt werden. «One of the most striking aspects in the clinical presentation of individuals with AN is the extreme degree to which they value and «defend their undernourished state» [Vitousek et al., 1998].

Im Bereich der Zwangsstörungen erfassten Külz et al. [2010] anhand einer Stichprobe stationärer Patienten die im Rahmen kognitiver Verhaltenstherapie erarbeiteten Funktionalitäten ihrer Symptomatik. Mithilfe inhaltsanalytischer Techniken konnte die Arbeitsgruppe 11 Funktionalitäten, die sich in die Kategorien «intraindividuell» und «interpersonell» einteilen lassen, identifizieren. Darauf aufbauend wurde zur quantitativen Erfassung von Kulla et al. [2015] der Fragebogen zu Funktionalitäten bei Zwangserkrankungen (FFZ) entwickelt. Die ermittelten Skalen decken sich größtenteils mit den von Külz et al. [2010] theoretisch postulierten Bereichen.

Auch bei Essstörungen ist davon auszugehen, dass es sich um «Lösungs-versuche für andere Schwierigkeiten, die primär in der Person und/oder primär in ihrem sozialen Bezugssystem liegen» [Lakatos und Reinecker, 1999, S. 52], handelt.
Vor dem Hintergrund des hohen Chronifizierungsrisikos und der hohen Relevanz der Bearbeitung der Funktionalität für den Therapieerfolg bzw. dessen Aufrechterhaltung bei Essstörungen scheint es von besonderer Bedeutung, diese eingehender zu betrachten. Das Ziel war es nun, mittels einer Pilotstudie die für die Patienten relevanten Funktionen bei Essstörungen zu erheben. Hierbei wurde ein patientenorientiertes Vorgehen gewählt.

\section{Methodik}

Fragestellungen

Das Ziel dieser Studie war es, Antworten auf die Fragen zu finden, welche Funktionalitäten ihrer Essstörung Patienten im Selbstbericht angeben, welche Funktionalitäten für die Patienten davon besonders relevant sind und ob es Unterschiede zwischen Patienten mit AN und Patienten mit BN hinsichtlich der Funktionalitäten gibt.

\section{Hypothesen}

Hypothese 1: Betroffene schreiben ihrer Essstörung häufiger intrapsychische als interpersonelle Funktionalitäten zu [Külz et al., 2010].

Hypothese 2: In Bezug auf die intrapsychischen Funktionalitäten stellen für Patientinnen mit AN Emotionsregulation, Sicherheit und Kontrolle sowie Inhalt und Struktur die wichtigsten Funktionalitäten dar, während für Patientinnen mit BN die Funktionalitäten Schönheitsideal und Emotionsregulation am bedeutendsten sind [Gale et al., 2006; Serpell et al., 2004; Serpell und Treasure, 2002; Serpell et al., 1999].

Hypothese 3: Es wird erwartet, dass für AN-Patientinnen die Funktionalitäten Sicherheit und Kontrolle, Inhalt und Struktur sowie Schutz vor Weiblichkeit/ Sexualität/dem Erwachsenwerden signifikant bedeutender sind als für ihre Vergleichsgruppe. Es wird erwartet, dass von BN-Patientinnen hingegen die Funktionalität Schönheitsideal als signifikant wichtiger bewertet wird [Gale et al., 2006].

Zusätzlich soll die Bedeutung interpersoneller Funktionalitäten explorativ untersucht werden.

Stichprobe mit Ein- und Ausschlusskriterien

Die Stichprobe umfasst 49 Frauen, die sich in einer stationären Behandlung in der Schön Klinik Roseneck in Prien am Chiemsee befanden.

Einschlusskriterien

Primärdiagnosen nach der International Classification of Diseases (ICD)-10: F50.0 (AN), F50.1 (atypische AN), F50.2 (BN) oder F50.3 (atypische BN), weibliches Geschlecht, ein Mindestalter von 18 Jahren, Einverständnis der Patientin.

\section{Ausschlusskriterien}

Männliches Geschlecht, Minderjährigkeit, akuter Substanzmissbrauch, akute Suizidalität, hirnorganische Erkrankung, das Vorliegen einer psychotischen oder bipolaren Störung, schwere lebensbedrohliche körperliche Erkrankungen, mangelnde Beherrschung der deutschen Sprache sowie fehlende Bereitschaft, an der Studie teilzunehmen bzw. fehlende Einwilligungserklärung.

\section{Erhebungsinstrumente}

Einleitend wurde eine Sichtung der Literatur zur Funktionalität von psychischen Störungen im Allgemeinen und Essstörungen im Speziellen und eine Sammlung der postulierten Funktionalitäten vorgenommen; zusätzliche, fehlende Aspekte wurden ergänzt. Im nächsten Schritt wurde ein Arbeitsblatt für Patienten zur Einschätzung der Funktionalitäten ihrer Essstörung erstellt.

Das eingesetzte Arbeitsblatt enthält einleitend eine offene Frage, die sehr allgemein gehalten ist: «Essstörungen können auch ihre positiven Seiten haben. Bitte überlegen Sie, wobei Ihnen die Essstörung helfen bzw. wofür sie gut sein könnte?» (Einleitungsfrage). Weiterhin wurden als Ergebnis der oben genannten Literaturrecherche 18 Funktionalitäten abgefragt. 11 Funktionalitäten wurden von Külz et al. [2010] übernommen; 7 essstörungsspezifische Kategorien wurden 
hinzugefügt [Fox et al., 2011; Gerlinghoff und Backmund, 2004; Higbed und Fox, 2010; Nordbø et al., 2006; Williams und Reid, 2010]. Die Probanden wurden hier jeweils gebeten, auf einer 5 -stufigen Likert-Skala $(1=$ Stimmt gar nicht bis $5=$ Stimmt völlig) einzuschätzen, inwieweit ihre Essstörung für sie die aufgeführte Funktion erfüllen würde. Die Abschlussfrage «Welche der obigen Aussagen beschreibt am treffendsten, welche Funktion die Essstörung in Ihrem Leben erfüllt?» bittet um Angabe der zutreffendsten Funktionalität.

Die Symptomschwere wurde anhand des Eating Disorder Inventory-2 (EDI 2) von Paul und Thiel [2005] ermittelt. Dies ist ein Instrument zur Erfassung von relevanten Verhaltensdimensionen bei essgestörten Patienten. Anhand von 91 Items werden Verhaltensweisen auf 11 Subskalen zusammengefasst: Schlankheitsstreben, Bulimie, Unzufriedenheit mit dem Körper, Ineffektivität, Perfektionismus, Misstrauen, Interozeptive Wahrnehmung, Angst vor dem Erwachsenwerden, Askese, Impulsregulation und Soziale Unsicherheit. Die interne Konsistenz der Skalen liegt in der Stichprobe der anorektischen und bulimischen Patienten zwischen 0,73 und 0,93. Die Ergebnisse der Test-Retest-Reliabilität liegen zwischen 0,81 und 0,89 . Das Instrument weist weiterhin gute Validitätsmaße auf [Paul und Thiel, 2005].

\section{Durchführung der Datenerhebung}

Das erstellte Arbeitsblatt wurde jeweils nach der 5. Sitzung der störungsspezifischen Gruppentherapie für Patientinnen mit Essstörungen, die das Thema «Funktionalität» behandelt, zusammen mit der Einverständniserklärung an die Probanden ausgegeben.

\section{Datenauswertung}

Die Antworten zu der offenen Einleitungsfrage des Arbeitsblattes wurden im ersten Schritt transkribiert und anschließend mittels der qualitativen Inhaltsanalyse nach Mayring [2003] systematisch gruppiert. Hierbei sind wir deduktiv vorgegangen [nach Mayring, 2002], d.h. es wurden die in der Literatur angegebenen Kategorien als Leitstrukturen angenommen. Tabelle 1 gibt Auskunft über die gefundenen Funktionalitäten. Die Zuordnung der Funktionalitäten zu den einzelnen Kategorien wurde anschließend durch 2 unabhängige Rater überprüft.

Im Folgenden werden deskriptive Statistiken berichtet. Zudem wurden Gruppenvergleiche mittels t-Tests durchgeführt.

\section{Ergebnisse}

\section{Stichprobe}

Es wurden 127 Patientinnen, die sich im Zeitraum von Januar bis Mai 2012 in stationärer Behandlung befanden und an der essstörungsspezifischen Gruppentherapie teilnahmen, um Studienteilnahme gebeten. 49 Patientinnen stimmten einer Teilnahme zu. Aufgrund von Minderjährigkeit $(\mathrm{n}=4)$, fehlender Einverständniserklärungen $(n=5)$ und Diagnosen, die außerhalb des Einschlussbereiches lagen $(n=3)$, wurden 12 Probandinnen aus den Analysen ausgeschlossen. Die Endstichprobe umfasste schließlich $37 \mathrm{~Pa}-$ tientinnen. Die eingeschlossenen Probandinnen unterschieden sich hinsichtlich Alter, Body-Mass-Index (BMI), Krankheitsdauer, Anzahl stationärer und ambulanter Vorbehandlungen sowie Verweildauer nicht signifikant von den Ausgeschlossenen.

Das Alter der eingeschlossenen Patientinnen lag zwischen 18 und 59 Jahren mit einem Mittel von 28,5 (Standardabweichung $(\mathrm{SD})=9,9)$ Jahren .

Von den Probandinnen wiesen 43,2\% die Diagnose F50.0 auf, 8,1\% die Diagnose F50.1, bei 43,2\% wurde die Diagnose F50.2 und bei 5,4\% die Diagnose 50.3 vergeben. Der Aufnahme-BMI bei Patientinnen mit der Erstdiagnose AN lag durchschnittlich bei $15,4 \mathrm{~kg} / \mathrm{m}^{2}$ $(\mathrm{SD}=2,2)$, bei den Patientinnen mit BN bei $21,7 \mathrm{~kg} / \mathrm{m}^{2}(\mathrm{SD}=4,6)$.
Bei 81,1\% der Patientinnen wurde eine komorbide Störung diagnostiziert. Zu den häufigsten komorbiden Diagnosen zählten dabei depressive Störungen (73,0\%), posttraumatische Belastungsstörungen (16,2\%), phobische Störungen (13,5\%) und Zwangsstörungen $(8,1 \%)$.

Die Verweildauer der Probandinnen betrug im Schnitt 97,6 (SD = 41,6) Tage. Die Patientinnen mit AN blieben durchschnittlich $114,8(\mathrm{SD}=46,3)$ Tage, die BN-Patientinnen 79,3 $(\mathrm{SD}=26,6)$ Tage. 91,9\% der Patientinnen schlossen ihren Aufenthalt regulär $a b, 2,7 \%$ brachen die Therapie auf eigenen Wunsch hin ab, 2,7\% wurden aus disziplinarischen Gründen entlassen. Diesbezügliche Angaben zu 1 Probandin fehlen.

Die Anzahl der stationären Vorbehandlungen lag durchschnittlich bei $1,3(\mathrm{SD}=1,6)$, die der ambulanten vorangegangenen Therapien bei $1,7(\mathrm{SD}=1,7)$.

Tabelle 2 gibt eine Übersicht über die demografischen Angaben sowie die Ergebnisse der Patientinnen hinsichtlich des EDI-2.

\section{Häufigkeitsverteilung der Funktionalitäten}

Abbildung 1 zeigt die Häufigkeitsverteilung der einzelnen Funktionalitäten nach den Ergebnissen aus der Einleitungs- und Abschlussfrage sowie aufgeteilt nach intrapsychischen und interpersonellen Kategorien. Insgesamt fanden sich die theoretisch angenommenen 18 Funktionalitäten wieder; 6 Freitextantworten, die weder einer bestehenden Kategorie zugeordnet noch aufgrund der lediglich vereinzelten Nennung als eigene Kategorie aufgeführt werden konnten, wurden vorerst der Kategorie Sonstige Funktionalitäten (z.B. Spargedanken) zugeteilt, letztlich aber nicht mit in die weiteren Analysen eingeschlossen. Sowohl in der Eingangs- als auch in der Abschlussfrage zählen Emotionsregulation sowie Sicherheit und Kontrolle zu den 3 am häufigsten genannten intrapsychischen Funktionalitäten. Nach der Einleitungsfrage zählt hierzu zudem Inhalt und Struktur, nach der Abschlussfrage Schönheitsideal. Diese Funktionalität wird in der Abschlussfrage mit $16,2 \%$ von den meisten Patientinnen als wichtigste intrapsychische Funktionalität gesehen, in der Einleitungsfrage wird Emotionsregulation (89,2\%) am häufigsten aufgeführt. Die intrapsychische Funktionalität Bewältigung einer anderen psychiatrischen Erkrankung wird in der Eingangsfrage hingegen am seltensten $(8,1 \%)$ benannt. Auf die Abschlussfrage hin wird diese intrapsychische Funktionalität, wie auch Selbstbestrafung, Inhalt und Struktur sowie Umgang mit hohen Leistungsanforderungen, von keiner Patientin als wichtigste Funktion gewählt. Die größte Diskrepanz in der Häufigkeit der Nennungen zwischen Einleitungsund Abschlussfrage findet sich für die Funktionalität Emotionsregulation.

Bei den interpersonellen Funktionalitäten werden in der Eingangsfrage folgende 3 Kategorien am häufigsten von den Patientinnen aufgeführt: Nähe und Geborgenheit (51,4\%), Abgrenzung/Ablösung/Autonomie (40,5\%) sowie Vermeidung von Anforderungen aus der Umwelt (37,8\%). Die erstgenannte Funktionalität wird auf die Abschlussfrage hin von keiner Probandin als am wichtigsten bewertet, wohingegen Abgrenzung/Ablösung/Autonomie (5,4\%) auch hier am zweithäufigsten benannt wird. Weiterhin werden 


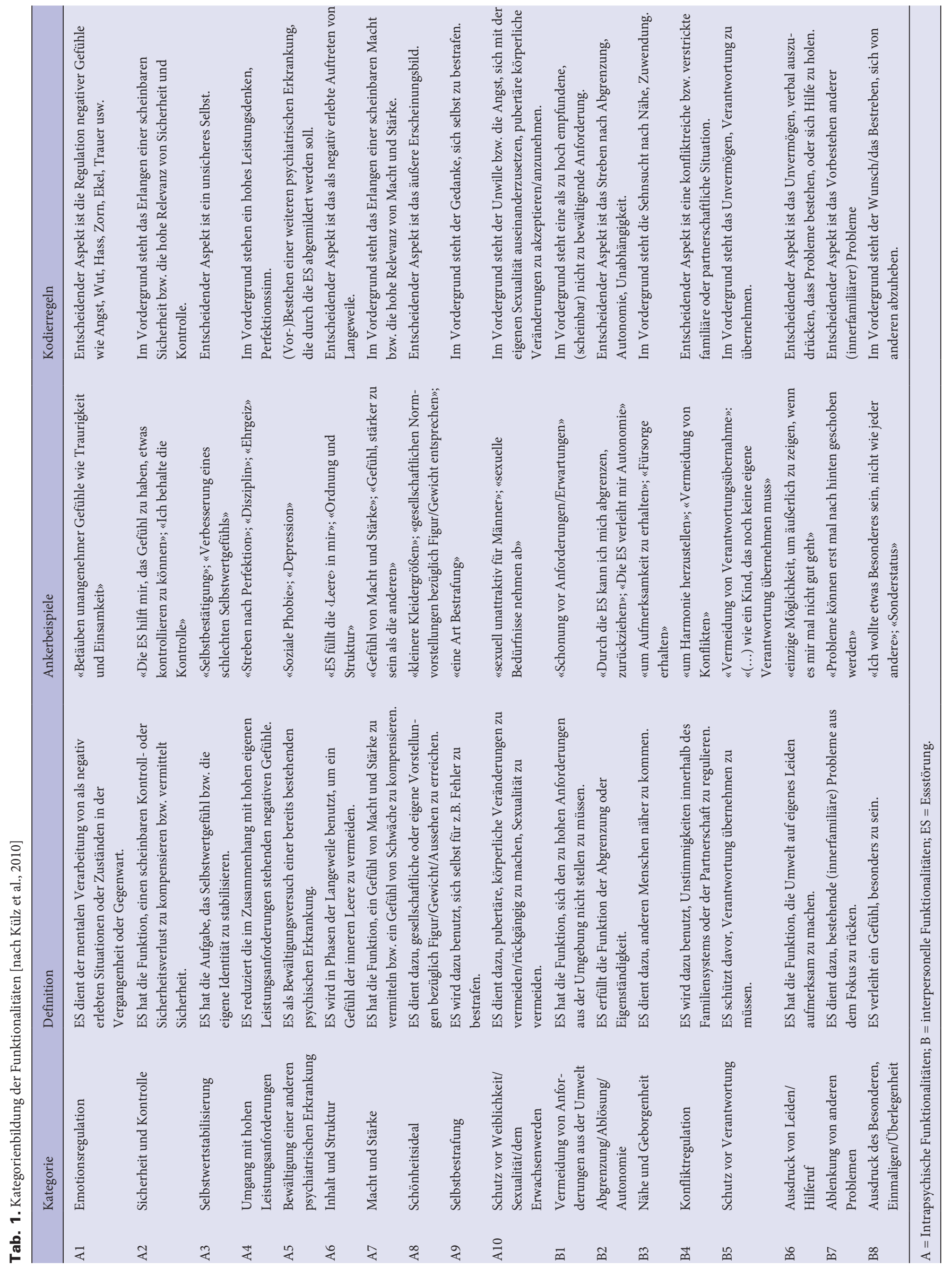


Tab. 2. Vergleich bezüglich Alter, BMI, Krankheitsdauer, Anzahl stationärer und ambulanter Vorbehandlungen und Verweildauer zwischen Patientinnen mit AN bzw. BN
Abb. 1. Häufigkeiten der intrapsychischen und interpersonellen Funktionalitäten.

\begin{tabular}{|c|c|c|c|c|c|}
\hline & $\begin{array}{l}\mathrm{AN}(\mathrm{N}=19) \\
\mathrm{M}(\mathrm{SD})\end{array}$ & $\begin{array}{l}\mathrm{BN}(\mathrm{N}=18), \\
\mathrm{M}(\mathrm{SD})\end{array}$ & $\mathrm{t}$ & df & $\mathrm{p}$ \\
\hline Alter, Jahre & $31,7(12,2)$ & $25,2(5,2)$ & 2,15 & 35 & 0,042 \\
\hline BMI bei Aufnahme, $\mathrm{kg} / \mathrm{m}^{2}$ & $15,4(2,2)$ & $21,7(4,6)$ & $-5,17$ & 34 & 0,000 \\
\hline Krankheitsdauer, Jahre & $8,6(8,4)$ & $8,8(5,6)$ & $-0,12$ & 34 & 0,908 \\
\hline Anzahl stationärer Vorbehandlungen, $\mathrm{n}$ & $1,8(1,9)$ & $0,8(1,0)$ & 1,85 & 34 & 0,075 \\
\hline Anzahl ambulanter Vorbehandlungen, $\mathrm{n}$ & $2,2(2,0)$ & $1,1(1,0)$ & 1,94 & 33 & 0,061 \\
\hline Verweildauer, Tage & $114,8(45,7)$ & $79,3(26,6)$ & 2,88 & 35 & 0,007 \\
\hline \multicolumn{6}{|l|}{ EDI-2 } \\
\hline Gesamtwert & $304,1(53,2)$ & $355,9(40,8)$ & $-3,18$ & 33 & 0,003 \\
\hline Schlankheitsstreben & $28,6(9,8)$ & $36,4(3,3)$ & $-3,27$ & 34 & 0,003 \\
\hline Bulimie & $14,0(5,9)$ & $31,2(7,1)$ & $-7,94$ & 34 & 0,000 \\
\hline Unzufriedenheit mit dem Körper & $39,5(9,2)$ & $45,8(7,6)$ & $-2,24$ & 34 & 0,032 \\
\hline Ineffektivität & $35,6(9,4)$ & $37,4(9,3)$ & $-0,57$ & 34 & 0,572 \\
\hline Perfektionismus & $23,5(5,6)$ & $23,8(5,0)$ & $-0,17$ & 34 & 0,869 \\
\hline Misstrauen & $23,3(5,6)$ & $25,5(6,6)$ & $-1,09$ & 34 & 0,283 \\
\hline Interozeptive Wahrnehmung & $33,9(9,1)$ & $41,5(7,5)$ & $-2,72$ & 34 & 0,010 \\
\hline Angst vor dem Erwachsenwerden & $25,0(6,4)$ & $26,9(7,2)$ & $-0,82$ & 33 & 0,421 \\
\hline Askese & $25,8(6,2)$ & $25,9(4,1)$ & $-0,02$ & 33 & 0,985 \\
\hline Impulsregulation & $28,4(8,7)$ & $32,4(9,0)$ & $-1,34$ & 33 & 0,189 \\
\hline Soziale Unsicherheit & $26,8(6,4)$ & $29,4(5,4)$ & $-1,25$ & 33 & 0,219 \\
\hline
\end{tabular}

$\mathrm{BMI}=$ Body Mass Index; $\mathrm{AN}=$ Anorexia nervosa BN = Bulimia nervosa; $\mathrm{M}=$ Mittelwert $\mathrm{SD}=$ Standardabweichung; EDI-2 = Eating Disorder Inventory-2.

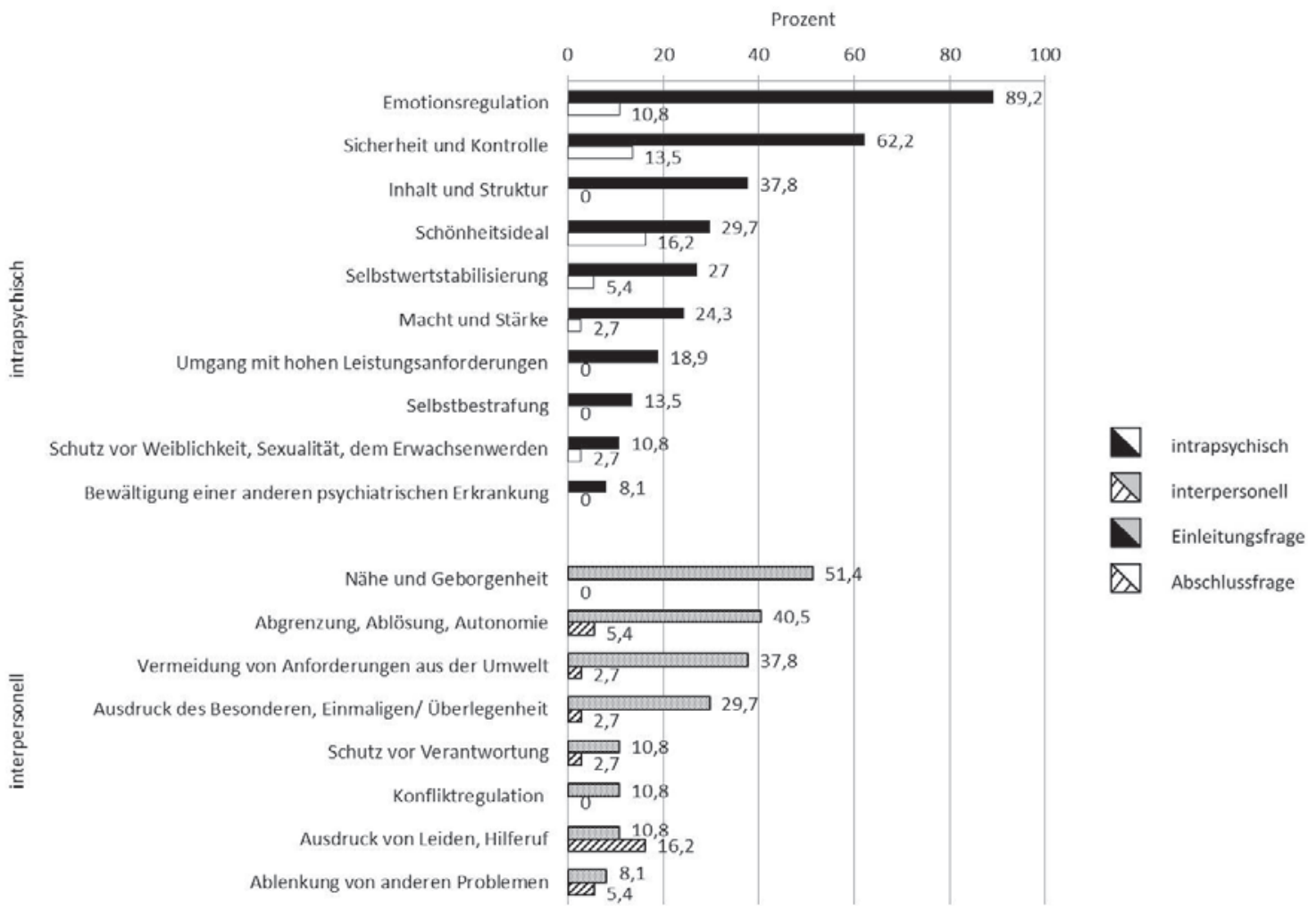

Ausdruck von Leiden/Hilferuf (16,2\%) sowie Ablenkung von anderen Problemen (5,4\%) am häufigsten aufgeführt.

Ein deutlicher Unterschied in der Häufigkeitsverteilung findet sich somit bei der Funktionalität Ausdruck von Leiden/Hilferuf, die bei den interpersonellen Funktionalitäten eingangs noch mit am seltensten benannt wird.
Bei Betrachtung der Übersicht insgesamt wird deutlich, dass die intrapsychischen im Vergleich zu den interpersonellen Funktionalitäten ausgeprägter sind. Während beispielsweise 35,1\% der Patientinnen auf die Abschlussfrage hin eine interpersonelle Funktionalität als am zutreffendsten auswählten, gaben 51,3\% eine intrapsychische Funktionalität an. 13,5\% der Patientinnen enthielten sich hier der Angabe. 


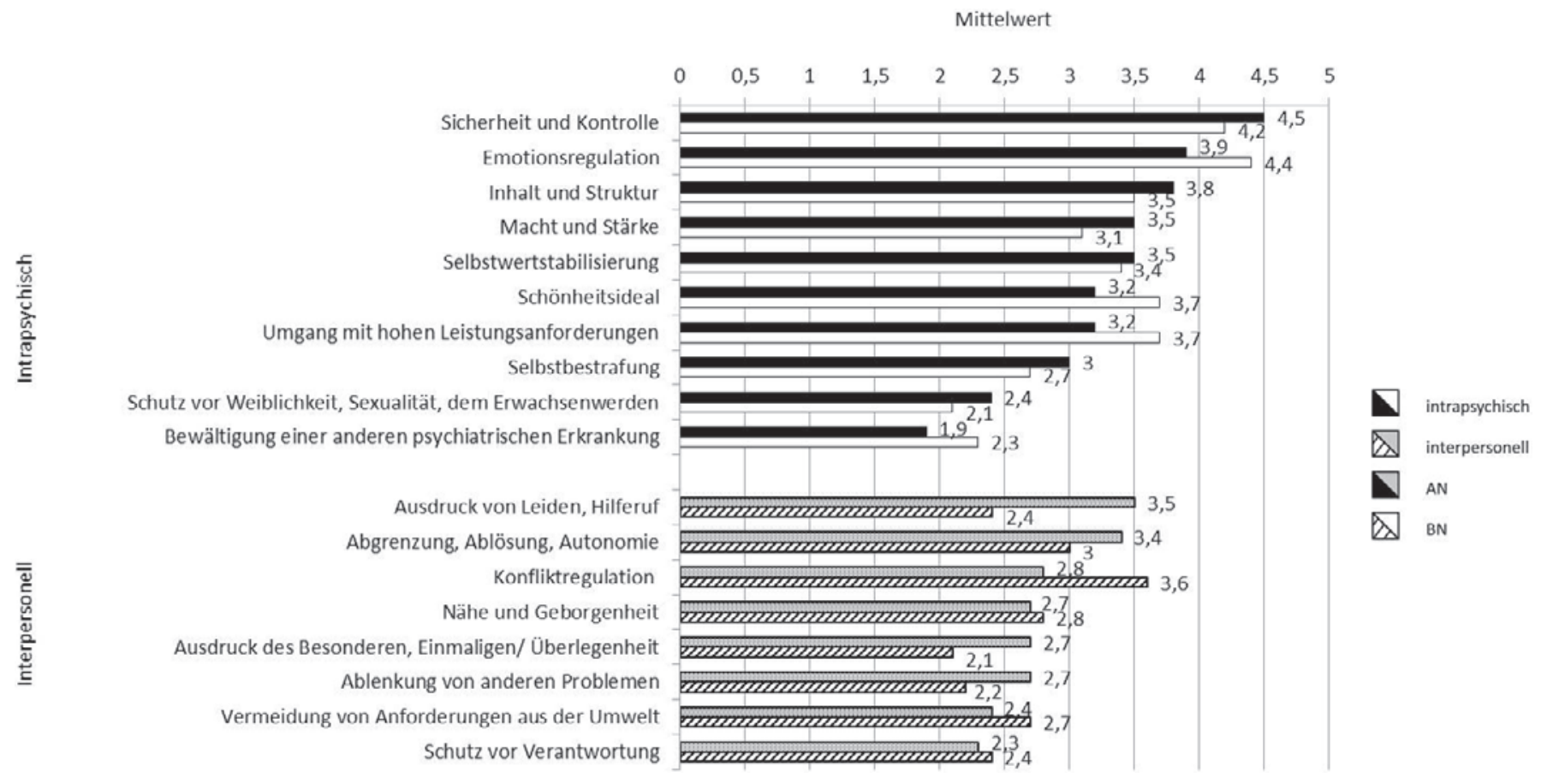

Abb. 2. Mittelwerte der intrapsychischen und interpersonellen Funktionalitäten.

\section{Mittelwerte der Skalen}

Bei den intrapsychischen Funktionalitäten weist Sicherheit und Kontrolle den höchsten Mittelwert auf $(\mathrm{M}=4,3 ; \mathrm{SD}=0,9)$, der niedrigste Mittelwert findet sich bei der Kategorie Bewältigung einer anderen psychiatrischen Erkrankung $(\mathrm{M}=2,1$; $\mathrm{SD}=1,6)$. Bei den interpersonellen Funktionalitäten wird den Funktionalitäten Konfliktregulation $(\mathrm{M}=3,2 ; \mathrm{SD}=1,6)$ wie auch Abgrenzung/Ablösung/Autonomie $(\mathrm{M}=3,2 ; \mathrm{SD}=1,5)$ im Mittel die höchste Relevanz zugeschrieben, Schutz vor Verantwortung erfährt hier die geringste Zustimmung $(\mathrm{M}=2,3 ; \mathrm{SD}=1,4)$.

\section{Vergleich: Qualitative und quantitative Auswertung}

Betrachtet man nun die Ergebnisse aus der qualitativen Analyse von Einleitungs- und Abschlussfrage mit den Daten der quantitativen Analyse, liegen die intrapsychischen Funktionalitäten Emotionsregulation sowie Sicherheit und Kontrolle sowohl bei der Auswertung der Antworten auf die Eingangs- und Abschlussfrage als auch nach quantitativer Auswertung unter den 3 relevantesten Funktionalitäten. Eine Übereinstimmung zwischen Eingangsfrage und quantitativer Auswertung findet sich auch bei Betrachtung der intrapsychischen Funktionalitäten, die seltener aufgeführt bzw. als weniger zutreffend bewertet wurden. Diese sind jeweils Bewältigung einer anderen psychiatrischen Erkrankung und Schutz vor Weiblichkeit/Sexualität/dem Erwachsenwerden.

Bei den interpersonellen Funktionalitäten ist die Kategorie $A b$ grenzung/Ablösung/Autonomie sowohl bei der quantitativen Auswertung als auch bei der Auswertung der Antworten auf die Eingangsund Abschlussfrage jeweils unter den 3 meistgenannten Funktionalitäten aufgelistet. Ausdruck von Leiden/Hilferuf ist hier bei der Abschlussfrage wie auch bei der quantitativen Auswertung zu finden. Weitere Übereinstimmungen oder Auffälligkeiten finden sich nicht.

\section{Vergleich $A N$ und $B N$}

Die Mittelwerte der einzelnen Funktionalitäten bei den Probandinnen mit AN im Vergleich zu den Probandinnen mit BN sind in Abbildung 2 dargestellt.

Ein signifikanter Unterschied zwischen den beiden Gruppen (AN: $\mathrm{M}=3,5, \mathrm{SD}=1,3$; $\mathrm{BN}: \mathrm{M}=2,4, \mathrm{SD}=1,7$ ) fand sich bei der interpersonellen Funktionalität Ausdruck von Leiden/Hilferuf $(\mathrm{t}(29)=$ $2,1 ; \mathrm{p}=0,04)$. So war die Zustimmung der Patientinnen mit AN in der Kategorie Ausdruck von Leiden/Hilferuf im Vergleich signifikant höher. Weitere signifikante Unterschiede ergaben sich nicht.

Abbildung 2 ist weiterhin Folgendes zu entnehmen: Deskriptiv stellen die wichtigsten intrapsychischen Funktionalitäten für die Patientinnen mit AN Kontrolle und Sicherheit, Emotionsregulation sowie Inhalt und Struktur dar, während die Patientinnen mit BN ebenso die Kategorien Emotionsregulation und Kontrolle und Sicherheit, daneben aber auch die Kategorien Schönheitsideal sowie Umgang mit hohen Leistungsanforderungen am höchsten bewertete.

Bei den interpersonellen Funktionalitäten erhielten Ausdruck von Leiden/Hilferuf, Abgrenzung/Ablösung/Autonomie und Konfliktregulation von den AN-Patientinnen die höchste Zustimmung. Die Probandinnen mit BN fanden sich in den Kategorien Konfliktregulation, Abgrenzung/Ablösung/Autonomie sowie Nähe und Geborgenheit am ehesten wieder.

\section{Korrelationen}

Es wurden weiterhin Zusammenhänge der Funktionalitäten mit den Subskalen des EDI-2, Alter, BMI bei Aufnahme, Verweildauer sowie Anzahl an ambulanten und stationären Vorbehandlungen in der Gesamtstichprobe untersucht. Auf eine Analyse getrennt für AN- und BN-Patientinnen wurde verzichtet, da sich wie oben dargestellt lediglich 1 signifikanter Unterschied hinsichtlich der Funk- 
Tab. 3. Signifikante Korrelationen (nach Pearson) der Funktionalitäten mit EDI-2-Subskalen, Alter, BMI, Krankheitsdauer und Anzahl ambulanter Vorbehandlungen

\begin{tabular}{ll}
\hline Funktionalität ${ }^{\mathrm{a}}$ & Korrelation \\
\hline Emotionsregulation & EDI-2 Schlankheitsstreben $(\mathrm{r}=0,481 ; \mathrm{p}=0,003)$ \\
Sicherheit und Kontrolle & BMI $(\mathrm{r}=-0,360 ; \mathrm{p}=0,031)$ \\
Bewältigung einer anderen & EDI-2 Misstrauen $(\mathrm{r}=0,425 ; \mathrm{p}=0,010)$ \\
\multicolumn{1}{c}{ psychiatrischen Erkrankung } & BMI $(\mathrm{r}=0,372 ; \mathrm{p}=0,026)$ \\
Macht und Stärke & Alter $(\mathrm{r}=-0,414 ; \mathrm{p}=0,011)$ \\
Schönheitsideal & EDI-2 Schlankheitsstreben $(\mathrm{r}=0,393 ; \mathrm{p}=0,018)$ \\
& EDI-2 Bulimie $(\mathrm{r}=0,340 ; \mathrm{p}=0,043)$ \\
& Krankheitsdauer $(\mathrm{r}=-0,405 ; \mathrm{p}=0,014)$ \\
& Alter $(\mathrm{r}=-0,441 ; \mathrm{p}=0,006)$ \\
Selbstbestrafung & ambulante Vorbehandlungen $(\mathrm{r}=0,382 ; \mathrm{p}=0,024)$ \\
Schutz vor Weiblichkeit/Sexualität/ & EDI-2 Unzufriedenheit mit dem Körper $(\mathrm{r}=0,0336 ; \mathrm{p}=0,049)$ \\
\multicolumn{1}{c}{ dem Erwachsenwerden } & EDI-2 Ineffektivität $(\mathrm{r}=0,412 ; \mathrm{p}=0,014)$ \\
Konfliktregulation & BMI $(\mathrm{r}=0,349 ; \mathrm{p}=0,037)$ \\
Schutz vor Verantwortung & EDI-2 Ineffektivität $(\mathrm{r}=0,352 ; \mathrm{p}=0,035)$ \\
& EDI-2 Angst vor dem Erwachsenwerden $(\mathrm{r}=0,529 ; \mathrm{p}=0,001)$ \\
& EDI-2 Soziale Unsicherheit $(\mathrm{r}=0,392 ; \mathrm{p}=0,020)$ \\
Ausdruck von Leiden/Hilferuf & Alter $(\mathrm{r}=0,373 ; \mathrm{p}=0,025)$ \\
Ablenkung von anderen Problemen & interozeptive Wahrnehmung $(\mathrm{r}=-0,357 ; \mathrm{p}=0,032)$ \\
\hline ED sind lediglich die Funktionalitäten aufgeführt, bei denen sich eine signifikante Korrelation fand. \\
\hline
\end{tabular}

tionalitäten gefunden hatte. In Tabelle 3 sind die signifikanten Korrelationen nach Pearson dargestellt. Als auf einem Niveau von 0,01 signifikant erwiesen sich die Korrelationen zwischen Emotionsregulation und EDI-2 Schlankheitsstreben, Bewältigung einer anderen psychiatrischen Erkrankung und EDI-2 Misstrauen, Schönheitsideal und Alter sowie Schutz vor Verantwortung und EDI-2 Angst vor dem Erwachsenwerden. Zusammenhänge der Funktionalitäten mit der Verweildauer wie auch mit der Anzahl an stationären Vorbehandlungen fanden sich nicht.

\section{Diskussion}

Ziel der vorliegenden Studie war es, Aufschluss über intrapsychische und interpersonelle Funktionalitäten von Essstörungen bei stationären Patienten zu gewinnen. Es ergaben sich 18 verschiedene Funktionalitäten. Zudem zeigte sich gemäß der Hypothese 1, dass intrapsychische im Vergleich zu interpersonellen Funktionalitäten sowohl im Selbstbericht als auch in der Abschlussfrage häufiger aufgeführt werden und auch durchschnittlich als zutreffender bewertet werden. Weiterhin sollte untersucht werden, welche intrapsychischen Funktionalitäten für die AN- bzw. BN-Patientinnen jeweils am bedeutendsten sind. Hier wurde vorab die Hypothese aufgestellt, dass für Patientinnen mit AN die wichtigsten intrapsychischen Funktionalitäten Emotionsregulation, Sicherheit und Kontrolle sowie Inhalt und Struktur darstellen, während für Patientinnen mit BN die Funktionalitäten Schönheitsideal und Emotionsregulation am bedeutendsten sind. Die Ergebnisse spiegeln diese Annahmen wider. Zusätzlich zu den in der Hypothese angenommenen Funktionalitäten bewerteten die BN-Patientinnen Kontrolle und Sicherheit sowie Umgang mit hohen Leistungsanforderungen als wichtig. Hypothese 3, in der erwartet wurde, dass sich Patientinnen mit
AN und BN hinsichtlich der subjektiv wahrgenommenen Funktionen ihrer Erkrankung unterscheiden, konnte bis auf den signifikanten Unterschied bei der Kategorie Ausdruck von Leiden/Hilferuf nicht bestätigt werden. Die untersuchten AN-Patientinnen erzielten hier signifikant höhere Mittelwerte als ihre Vergleichsgruppe.

Wie bei den Untersuchungen zu Funktionalitäten bei Zwangsstörungen [Kulla et al., 2015; Külz et al., 2010] berichteten so also auch die befragten Essstörungspatientinnen eine Vielzahl an Funktionalitäten ihres Störungsbildes. Wie dargestellt finden sich alle vorab auf Basis einer Literaturrecherche erhobenen Funktionalitäten ebenso im Selbstbericht der Probanden. Sowohl Patientinnen mit BN als auch Patientinnen mit AN scheinen einen wichtigen Nutzen ihrer Störung darin zu sehen, mit unangenehmen Gefühlen umzugehen. Dies fanden auch Gale et al. [2006].

Betroffene von AN sehen die wichtigste Funktion der Störung darin, dass sie ihnen Sicherheit und Kontrolle vermittelt. Auch in der Untersuchung von Gale et al. [2006] zeigte sich die Wichtigkeit dieser Funktionalität für AN-Patientinnen. Weiterhin fanden sich AN-Patientinnen wie bei Gale et al. [2006] häufig in der Kategorie Inhalt und Struktur wieder.

Es fällt zudem auf, dass BN-Patientinnen ihrer Essstörung stärker die Funktionalität Schönheitsideal zuschreiben. Auch bei Gale et al. [2006] zeigte sich, dass BN-Betroffene einen Vorteil ihrer Erkrankung darin sehen, essen zu können und dennoch schlank zu bleiben («Eat but Stay Slim»). Sie unterschieden sich hier signifikant von der Vergleichsgruppe der AN-Patientinnen.

Im Gegensatz zu den Ergebnissen der vorliegenden Studie fanden sich bei Gale et al. [2006] insgesamt 5 signifikante Unterschiede zwischen AN- und BN-Patienten. Dass sich die Vergleichsgruppen in unserer Untersuchung lediglich hinsichtlich der Funktionalität Ausdruck von Leiden/Hilferuf signifikant voneinander unterscheiden, könnte einerseits auf die kleine Fallzahl zurückzuführen sein. 
Ein anderer Ansatz der Interpretation, weshalb sich beim Vergleich der AN- und BN-Probandinnen nur 1 signifikanter Unterschied fand, kann eventuell auch die transdiagnostische Sichtweise [vgl. Cooper und Shafran, 2008; Fairburn et al., 2003] sein. Diese weicht die Grenzen zwischen den Störungsbildern auf und betrachtet sie aufgrund der postulierten gemeinsamen Psychopathologie als Phasen einer Erkrankung [Fairburn et al., 2003], d.h. als unterschiedliche Ausprägungen einer Dimension. Ob sich AN- und BN-Patientinnen in ihren Funktionalitäten unterscheiden, sollte deshalb in zukünftigen Studien noch einmal näher beleuchtet werden.

Es zeigte sich, dass die Funktionalität Emotionsregulation und die EDI-2-Subskala Schlankheitsstreben signifikant miteinander korrelieren. Auch andere Studien, die den Zusammenhang zwischen Defiziten in der Emotionsregulation und Essstörungen untersuchten, fanden signifikante Korrelationen [vgl. Harrison et al., 2010]. Ein eindeutiger Zusammenhang ergab sich auch zwischen Schutz vor Verantwortung und der EDI-2-Subskala Angst vor dem Erwachsenwerden sowie eine negative Korrelation zwischen Schönheitsideal und dem Alter der Probandinnen. Letztgenannter $\mathrm{Zu}$ sammenhang weist in die Richtung, dass bei älteren Essstörungspatientinnen weniger (oder nicht mehr) das Streben nach einem Figurideal, sondern vielmehr andere Funktionalitäten eine Rolle spielen. Es zeigte sich zudem, dass ebenso die Funktionalitäten Macht und Stärke für ältere Patientinnen von untergeordneter Bedeutung, Ausdruck von Leiden/Hilferuf hingegen von verstärkter Bedeutung sind.

Interessant ist vor allem auch die Betrachtung der Ergebnisse des Selbstberichtes im Vergleich zur Einschätzung der zutreffendsten Funktionalität am Ende der Befragung. Bei den intrapsychischen Funktionalitäten werden hier jeweils Emotionsregulation sowie Sicherheit und Kontrolle benannt. Dies spricht dafür, dass die Patientinnen sich darüber bewusst sind, in welcher Hinsicht sie vor allem von ihren Symptomen profitieren. Bei der Kategorie Emotionsregulation findet sich allerdings die größte Diskrepanz, wie häufig eine Funktionalität jeweils in der Einleitungs- und Abschlussfrage aufgeführt wird, über die gesamten Kategorien hinweg. Bei den interpersonellen Funktionalitäten fällt zudem auf, dass die Funktionalität Ausdruck von Leiden/Hilferuf, die abschließend am häufigsten benannt wird, auf die Eingangsfrage hin noch mit am seltensten aufgeführt wird. Daraus lässt sich folgern, dass für die Patientinnen bestimmte Funktionalitäten offensichtlich sind und sie sich dieser bewusst sind. Andere erscheinen unbewusst oder nicht spontan abrufbar; werden die Patientinnen jedoch darauf hingewiesen, erkennen sie deren Relevanz.

Diese Kombination aus qualitativer und quantitativer Datengewinnung ist als Stärke der vorliegenden Untersuchung zu sehen. Die qualitativen Daten ermöglichten es uns, individuelle Spontannennungen zu erfassen. Durch die zusätzliche quantitative Erhebung wurde eine höhere Vergleichbarkeit der Ergebnisse erzielt. Weiterhin konnten auch Funktionalitäten erfasst werden, die von den Teilnehmerinnen nicht spontan genannt und möglicherweise bislang auch nicht reflektiert wurden. Die Untersuchung sowohl von $\mathrm{AN}$ - als auch von BN-Patientinnen machte weiterhin einen Vergleich der beiden Störungsbilder hinsichtlich der Funktionalitäten möglich.
Es ist jedoch darauf zu verweisen, dass die Generalisierbarkeit der Ergebnisse dieser Studie aufgrund der bereits angesprochenen kleinen Probandenzahl eingeschränkt ist. Weiterhin handelt es sich bei der Stichprobe um stationäre Patientinnen einer psychosomatischen Klinik, weshalb ein Rückschluss auf andere Patientengruppen nur bedingt möglich ist. Untersuchungen unter Einbezug psychiatrischer und ambulanter Patienten wären somit interessant. Weiterhin ist hier anzumerken, dass die Routinediagnosen nicht vor Einschluss der Probandinnen mittels SKID (Strukturiertes Klinisches Interview für DSM-IV) [Wittchen et al., 1997] oder essstörungsspezifischer Diagnoseinstrumente wie beispielsweise SIAB (Strukturiertes Inventar für Anorektische und Bulimische Essstörungen nach DSM-IV und ICD-10) [Fichter und Quadflieg, 2001] überprüft wurden.

Einschränkend ist weiterhin zu sehen, dass lediglich die subjektive Patientensicht einbezogen wurde, die Meinung der Therapeuten blieb unberücksichtigt. Subjektive Krankheitsmodelle haben sich als entscheidend für die Prognose des Therapie- und Krankheitsverlaufs erwiesen, können aber von den (objektiven) Ursachen abweichen [Petrie und Weinman, 1997]. Zukünftige Untersuchungen sollten die Funktionalitäten auch aus Therapeutensicht erheben, um eine Einschätzung dafür zu bekommen, inwieweit die Funktionalität der Essstörungen aus Patienten- und Therapeutensicht übereinstimmt. Weiterhin wäre es interessant, die Veränderung der Funktionalitäten über den Therapieverlauf hinweg zu betrachten; Erhebungen sollen in einer Folgestudie deshalb zu mehreren unterschiedlichen Messzeitpunkten stattfinden. So lässt sich in der vorliegenden Erhebung auch nicht ausschließen, dass sich die subjektive Einschätzung der Funktionalitäten im Rahmen der zu diesem Zeitpunkt bereits erfolgten therapeutischen Interventionen bereits verändert hat.

Neben essstörungsspezifischen Funktionalitäten wie z.B. Schönheitsideal zeigten sich bei den AN- und BN-Patientinnen auch Funktionalitäten, die bei Patienten mit Zwangsstörungen gefunden wurden [Külz et al., 2010]. Wie Zwangserkrankte schreiben die Probandinnen dieser Studie ihrer Essstörung sowohl häufiger als auch mit höherer Relevanz eine intrapsychische Funktionalität zu. Am häufigsten wird im Speziellen Emotionsregulation benannt. Weiterhin dient den Essstörungspatientinnen wie auch den Patienten mit einer Zwangsstörung ihre Erkrankung der Bewältigung hoher Sicherheits- und Kontrollbedürfnisse. Bedeutend sind für beide Störungsbilder auch die Funktionalitäten Selbstwertregulation sowie Abgrenzung/Ablösung/Autonomie. Dies deutet darauf hin, dass es sich bei Funktionalitäten nicht ausschließlich um störungsspezifische Themen handelt. In einer zukünftigen Studie wäre es somit interessant, sich mit der Spezifität der Funktionalitäten zu befassen.

Die Ergebnisse könnten Implikationen für die störungsspezifische Psychotherapie haben. Die Bandbreite an berichteten Funktionalitäten zeigt so wiederum die Bedeutung der Er- und Bearbeitung dieser auf - nicht allein für die Symptomreduktion, sondern aus einer übergeordneten Perspektive betrachtet als eine subjektive Entlastung für die Patienten. Diese Entlastung können sie beispielsweise durch den Gewinn eines Verständnisses für die Sinnhaftigkeit ihrer Symptomatik und durch das Gefühl, von ihrem 
Therapeuten auch im Gesamtkontext ihrer Lebenssituation wahrgenommen zu werden, erfahren. Weiterhin können Funktionalitäten Anhaltspunkte für die Definition von störungsübergreifenden Therapiezielen liefern, die für die Lebensgestaltung der Betroffenen von entscheidender Bedeutung sein können. Da die psychischen Symptome im Verlauf für die Betroffenen bestimmte Aufgaben im Umgang mit unangenehmen intrapsychischen Zuständen oder mit ihrer Umwelt übernommen haben, stellt sich immer die Frage nach alternativen Bewältigungsstrategien, die es im Rahmen der Therapie zu erarbeiten gilt. Dies erscheint vor allem unter dem Aspekt relevant, dass nicht bearbeitete Funktionalitäten Risikofaktoren für Non-Response und somit für Chronifizierung darstellen. Um nach der Offenlegung der individuellen Funktionalitäten die Therapiemotivation gezielt zu fördern, wäre der Einsatz des Motivational Interviewing denkbar. Studien, die diese Technik an Essstörungspatientinnen entweder im persönlichen Gespräch oder onlinebasiert eingesetzt untersuchten, berichten Erfolge [Hötzel et al., 2013; Weiss et al., 2013]. Um noch zielgerichteter vorgehen $\mathrm{zu}$ können, wäre es hier allerdings auch interessant zu untersuchen, bei welchen Patienten die Betrachtung von Funktionalitäten unabdingbar ist und bei welchen sie weniger zentral ist, um die Symptomatik zu reduzieren.

Die Ergebnisse zeigen auf, dass die Vermittlung von Fertigkeiten im Emotionsmanagement ein zentraler Bestandteil der Therapie sein sollte, vor allem bei Patienten mit BN. Hier sei auch auf die hohe Komorbidität der BN mit der Borderline-Persönlichkeitsstörung verwiesen [vgl. Sansone et al., 2005]. Neuere Therapiemethoden wie die Dialektisch Behaviorale Therapie bei Essstörungen [Kröger et al., 2010] oder emotionsfokussierte Ansätze [vgl. Dolhanty und Greenberg, 2009] legen hierauf ein Augenmerk.

Das Ergebnis, dass die Funktionalität Schönheitsideal vor allem für BN-Patientinnen zu den 3 wichtigsten Kategorien gehört, bestätigt die Annahme, dass spezielle, auf das Störungsbild zugeschnittene Therapieelemente integraler Bestandteil der Behandlung von Essstörungen sein sollten. Kernthema sollte hier Körperakzeptanz sein, wie dies z.B. bei der Körperbildtherapie [Vocks und Legenbauer, 2010] der Fall ist.

Aber nicht allein für die Therapie, auch für die Prävention von Essstörungen kann das Wissen um eventuelle Funktionalitäten von
Bedeutung sein. So könnte beispielsweise die gezielte Förderung des Emotionsmanagements oder aber des Selbstwertes bereits Bestandteil von Präventionsmaßnahmen sein.

Mit der Pros and Cons of Eating Disorders-Skala (P-CED) von Gale et al. [2006] wurde bereits ein Instrument vorgelegt, um Vorund Nachteile von Essstörungen zu erfassen. Die vorliegende Untersuchung beschäftigte sich ausschließlich mit den subjektiv wahrgenommenen «Pros» einer Essstörung, da diese aus Sicht der Autoren einerseits schwerer zu erfassen sind, da sie schwieriger zugänglich sind, ihnen andererseits aber bezüglich der Aufrechterhaltung der Essstörung eine größere Bedeutung zukommt als den Nachteilen und sie somit als therapierelevanter erachtet werden. Dabei wurde versucht, die Vorteile einer Essstörung noch spezifischer zu erfassen, indem sowohl intrapsychische als auch interpersonelle Funktionalitäten abgefragt wurden. Im Rahmen der P-CED wurden bisher nur intrapsychische Funktionalitäten berücksichtigt.

Ziel wird es nun sein, auf Basis dieser Ergebnisse einen Fragebogen $\mathrm{zu}$ entwickeln, um die Ergebnisse nochmals detaillierter auf quantitativer Ebene erfassen zu können. Dieser soll anhand einer Stichprobe von 200 stationären Patientinnen validiert werden. Mithilfe des Fragebogens sollen die Funktionalitäten zu unterschiedlichen Messzeitpunkten erhoben werden, um Veränderungen im Therapieverlauf zu erfassen. Zudem sollen mögliche Zusammenhänge zwischen den Funktionalitäten und dem Behandlungserfolg betrachtet werden. Mittels einer Halbjahreskatamnese wird hier auch dessen Aufrechterhaltung über einen längeren Zeitraum untersucht werden.

Dieses Instrument soll es künftig ermöglichen, die von den Patienten subjektiv wahrgenommenen Funktionalitäten systematisch und zeiteffektiv zu erfassen, um sie dann in der Therapie gezielt bearbeiten zu können. Weiterhin soll es Patienten Hilfestellung und Anregung bieten, sich neben den negativen Konsequenzen der Erkrankung damit auseinanderzusetzen, welchen subjektiven «Gewinn» sie aus ihrer Essstörung ziehen.

\section{Disclosure Statement}

Es bestehen keine Interessenkonflikte.

\section{Literatur}

von Brachel R, Hirschfeld G, Hötzel K, Dörries A, Striegler K, Braks K, Huber TJ, Vocks S: Measuring the benefits and burdens of anorexia and bulimia nervosa - the German version of the Pros and Cons of Eating Disorders scale. Eingereicht.

Cooper Z, Shafran R: Cognitive behaviour therapy for eating disorders. Behav Cogn Psychother 2008;36:713722.

Dolhanty J, Greenberg LS: Emotion-focused therapy in a case of anorexia nervosa. Clin Psychol Psychother 2009;16:366-382.

DWDS: Das digitale Wörterbuch der deutschen Sprache des 20. Jh. Berlin-Brandenburgische Akademie der Wissenschaften, $w w w . d w d s . d e, 2003$.

Fairburn CG: Cognitive Behavior Therapy and Eating Disorders. New York, Guilford Press, 2008.
Fairburn CG: Kognitive Verhaltenstherapie und Essstörungen. Stuttgart, Schattauer, 2012.

Fairburn CG, Cooper Z, Shafran R: Cognitive behaviour therapy for eating disorders: a 'transdiagnostic' theory and treatment. Behav Res Ther 2003;41:509-528.

Fichter M, Quadflieg N: Das Strukturierte Interview für Anorektische und Bulimische Ess-Störungen nach DSM-IV und ICD-10 zur Expertenbeurteilung (SIABEX) und dazugehöriger Fragebogen zur Selbsteinschätzung (SIAB-S). Verhaltenstherapie 2001;11:314-325.

Fox AP, Larkin M, Leung N: The personal meaning of eating disorder symptoms: an interpretative phenomenological analysis. J Health Psychol 2011;16:116-125.

Friederich HC, Herzog W, Wild B, Zipfel S, Schauenburg $\mathrm{H}$ : Anorexia nervosa. Fokale psychodynamische Psychotherapie. Göttingen, Hogrefe, 2014.
Gale C, Holliday J, Troop NA, Serpell L, Treasure J: The pros and cons of change in individuals with eating disorders: a broader perspective. Int J Eat Disord 2006;39: 394-403.

Garner DM, Bemis KM: A cognitive-behavioral approach to anorexia nervosa. Cognit Ther Res 1982;6:123-150.

Geller J, Cockell SJ, Drab DL: Assessing readiness for change in the eating disorders: the psychometric properties of the readiness and motivation interview. Psychol Assess 2001;13:189.

Gerlinghoff M, Backmund H: Wege aus der Essstörung, ed 4. Stuttgart, Trias, 2004.

Hand I: Systemische Aspekte in der Verhaltenstherapie von Zwangsstörungen; in Ecker W (Hrsg): Die Behandlung von Zwängen: Perspektiven für die klinische Praxis. Bern, Huber, 2002, pp 81-100. 
Harrison A, Sullivan S, Tchanturia K, Treasure J: Emotional functioning in eating disorders: attentional bias, emotion recognition and emotion regulation. Psychol Med 2010;40:1887-1897.

Higbed L, Fox JR: Illness perceptions in anorexia nervosa: a qualitative investigation. Br J Clin Psychol 2010;49:307-325.

- Hötzel K, von Brachel R, Schmidt U, Rieger E, Kosfelder J, Hechler T, Schulte D, Vocks S: An internet-based program to enhance motivation to change in females with symptoms of an eating disorder: a randomized controlled trial. Psychol Med 2013;1-17.

Kröger C, Schweiger U, Sipos V, Kliem S, Arnold R, Schunert T, Reinecker H: Dialectical behaviour therapy and an added cognitive behavioural treatment module for eating disorders in women with borderline personality disorder and anorexia nervosa or bulimia nervosa who failed to respond to previous treatments. An open trial with a 15-month follow-up. J Behav Ther Exp Psychiatry 2010;41:381-388.

Kulla P, Schlegl S, Katrin Külz A, Förstner U, Warschburger P, Voderholzer U: Funktionalitäten von Zwangsstörungen - Entwicklung und Validierung eines Fragebogens (FFZ). Psychother Psych Med 2015;65:213-222.

Külz AK: Die Funktionsanalyse in der Verhaltenstherapie. Verhaltenstherapie 2014;24:211-220.

Külz AK, Lumpp A, Herbst N, Stelzer N, Förstner U, Voderholzer U: Welche Funktionen erfüllen Zwangssymptome? - Ergebnisse einer Analyse im stationären Setting. Verhaltenstherapie 2010;20:101-108.

Lakatos A, Reinecker H: Kognitive Verhaltenstherapie bei Zwangsstörungen. Ein Therapiemanual. Göttingen, Hogrefe, 1999.

Mayring P: Einführung in die qualitative Sozialforschung, ed 5. Weinheim, Beltz, 2002.

Mayring P: Qualitative Inhaltsanalyse: Grundlagen und Techniken, ed 8. Weinheim, Beltz, 2003.

Mentzos S: Lehrbuch der Psychodynamik: Die Funktion der Dysfunktionalität psychischer Störungen, ed 2. Göttingen, Vandenhoeck und Ruprecht, 2009.
Nordbø RHS, Espeset EMS, Gulliksen KS, Skårderud F, Holte A: The meaning of self-starvation: qualitative study of patients' perception of anorexia nervosa. Int J Eat Disord 2006;39:556-564.

Owens RG, Ashcroft JB: Functional analysis in applied psychology. Br J Clin Psychol 1982;21:181-189.

Paul T, Thiel A: Eating Disorder Inventory-2 (EDI-2): Deutsche Version. Göttingen, Hogrefe, 2005.

Petrie KJ, Weinman JA: Perceptions of Health and Illness. Current Research and Applications. Amsterdam, OPA, 1997.

Salbach-Andrae H, Jacobi C, Jaite C: Anorexia und Bulimia nervosa im Jugendalter. Kognitiv-verhaltenstherapeutisches Behandlungsmanual. Weinheim, Beltz, 2010.

Sansone RA, Levitt JL, Sansone LA: The prevalence of personality disorders among those with eating disorders. Eat Disord 2005;13:7-21

Schmidt U, Treasure J: Anorexia nervosa: valued and visible. A cognitive-interpersonal maintenance model and its implications for research and practice. Br J Clin Psychol 2006;45:343-366.

Schmidt U, Wade TD, Treasure J: The Maudsley Model of Anorexia Nervosa Treatment for Adults (MANTRA): development, key features, and preliminary evidence. J Cognit Psychother 2014;28:48-71.

Serpell L, Neiderman M, Haworth E, Emmanueli F, Lask B: The use of the Pros and Cons of Anorexia Nervosa (PCAN) scale with children and adolescents. J Psychosom Res 2003;54:567-571.

Serpell L, Teasdale JD, Troop NA, Treasure J: The development of the P-CAN, a measure to operationalize the pros and cons of anorexia nervosa. Int J Eat Disord 2004;36:416-433.

Serpell L, Treasure J: Bulimia nervosa: friend or foe? The pros and cons of bulimia nervosa. Int J Eat Disord 2002;32:164-170.

Serpell L, Treasure JD, Teasdale J, Sullivan V: Anorexia nervosa: friend or foe? Int J Eat Disord 1999;25:177-186.
Smink F, van Hoeken D, Hoek H: Epidemiology, course, and outcome of eating disorders. Curr Opin Psychiatry 2013;26:543-548.

Treasure J, Schmidt U: Ready, willing and able to change: motivational aspects of the assessment and treatment of eating disorders. Eur Eat Disord Rev 2001;9:4-18.

Treasure J, Schmidt U: The cognitive-interpersonal maintenance model of anorexia nervosa revisited: a summary of the evidence for cognitive, socio-emotional and interpersonal predisposing and perpetuating factors. J Eat Disord 2013;1:13-23.

Victorian Government Department of Human Services: The Victorian Burden of Disease Study: Mortality and morbidity in 2001. www2.health.vic.gov.au/getfile/ ?sc_itemid $=\% 7 B F 84 D 614 F-47 F C-4027-B D 0 B-$ 777AA49070A7\%7Detitle=Victorian\%20Burden\%20 of\%20Disease\%20Study, 2011.

Vitousek K, Ewald LS: Self-representation in eating disorders: a cognitive perspective; in Segal ZV, Blatt SJ (Hrsg): The Self in Emotional Distress: Cognitive and Psychodynamic Perspectives. New York, Guilford Press, 1993, pp 221-257.

Vitousek K, Watson S, Wilson G: Enhancing motivation for change in treatment-resistant eating disorders. Clin Psychol Rev 1998;18:391-420.

Vocks S, Legenbauer T: Körperbildtherapie bei Anorexia und Bulimia nervosa: Ein kognitiv-verhaltenstherapeutisches Behandlungsprogramm, ed 2. Göttingen, Hogrefe, 2010.

Weiss CV, Mills JS, Westra HA, Carter JC: A preliminary study of motivational interviewing as a prelude to intensive treatment for an eating disorder. J Eat Disord 2013;1:34.

Williams S, Reid M: Understanding the experience of ambivalence in anorexia nervosa: the maintainer's perspective. Psychol Health 2010;25:551-567.

Wittchen HU, Zaudig M, Fydrich T: Strukturiertes Klinisches Interview für DSM IV. Achse I und II. Göttingen, Hogrefe, 1997 